\title{
Airborne Spiders Drift on Multiple Silk Threads
}

\author{
Simulations reveal new details of the way spiders can fly by exploiting the \\ electric field present in the atmosphere.
}

By Rachel Berkowitz

$\square$ ome spiders can float and drift like a balloon, with lift provided by electrostatic forces or air currents or both. Now researchers have simulated the process at a new level of detail and have shown the importance of the spatial arrangement of the threads that the spider emits and uses to fly [1]. In contrast to earlier models that involve only a single thread and some subset of environmental forces, the new simulations provide a complete description of the force balance that determines whether a spider balloons or stays on the ground.

Explanations for spiders' ability to float fall into two main categories. Both invoke forces that act on the silk threads a

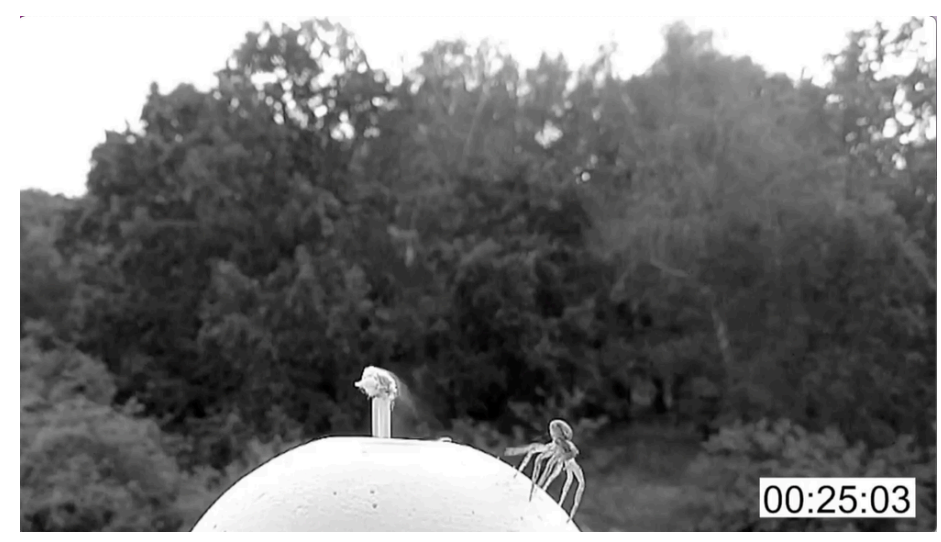

This crab spider (genus Xysticus) emits threads that allow it to launch into the air and float away. This is one of the larger ballooning spiders, with legs spreading across an area about $1 \mathrm{~cm}$ wide, in this case.

Credit: Plos Biol. 16, e2004405 (2018) (CC BY 4.0) spider emits for this purpose, which can number in the hundreds, depending on the species. One idea is that when air warmed by the sun rises, the spider's threads catch the updraft. The other hypothesis is that the atmosphere's weak but ever-present vertical electric field acts on the threads' static electric charge.

Researchers have previously explored both hypotheses with experiments, but it's difficult to observe the effects of airflow or electric fields on tiny, wispy threads. Simulations have been performed in one dimension and have assumed a single thread, even though multiple threads can have significant effects, says M. Khalid Jawed of the University of California, Los Angeles. For example, in the electrostatic mechanism, the lift force should be strongly affected by the spatial distribution of charge, which is determined by the threads' configuration in space.

Now, Jawed and Charbel Habchi of Notre Dame University-Louaize in Lebanon have turned to an algorithm developed by the computer graphics community. "Several hit Hollywood movies, such as The Hobbit and the Planet of the Apes series, have used this formulation for fur and hair," Habchi says.

The algorithm divides each thread into many spaghetti-like segments that can bend, stretch, and twist. The simulations approximate an Erigone spider as a 2-mm-wide solid sphere with 2, 4, or 8 threads attached at the top, oriented vertically at the start. Each thread is coated with electric charges. The researchers accounted for gravity; the atmosphere's electric field, which decreases with height; the threads' electric charge; and air resistance (drag) on the threads. 


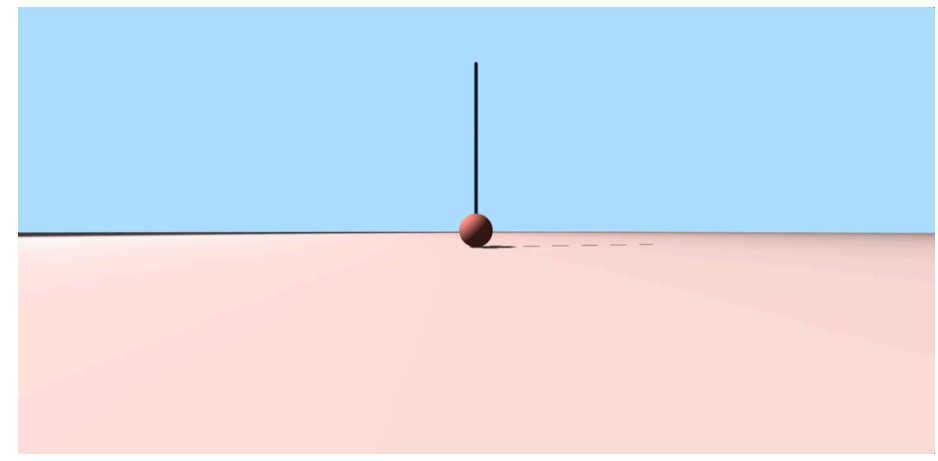

Simulations show how silk threads extending from a spider's abdomen spread out. The charged threads repel one another, and the spatial configuration of the threads influences the aerodynamic drag they experience.

Credit: C. Habchi and M. K. Jawed [1]

In the simulations, the spider starts at rest on the ground and is lifted by the electric field. While the charged, initially straight threads remain attached to the spider, their mutual repulsion causes them to spread apart over a period of time. As the spider accelerates upward, downward drag increases and-combined with the spider's weight-eventually cancels the lifting force. This competition between upward and downward forces determines a spider's final (terminal) upward velocity.

The researchers conclude that multiple threads, if charged, will retain an ordered arrangement rather than tangling, as they might in the absence of any charge or electric field. "We think that, at least for small spiders, the electric field, without any help from upward air currents, can cause ballooning," says Habchi. However, larger spiders would require a boost from upward airflow.

Habchi and Jawed found that their typical computed vertical velocities of $8.5 \mathrm{~cm} / \mathrm{s}$ agreed with recent experimental studies of Erigone spiders enclosed in a chamber with a controllable electric field. They hope to observe ballooning in natural spider habitats while measuring the atmospheric charge, wind, and other environmental factors.
Peter Gorham, a particle astrophysicist at the University of Hawaii who also studies spiders, says that the researchers "have tackled the mathematics associated with this complex classical

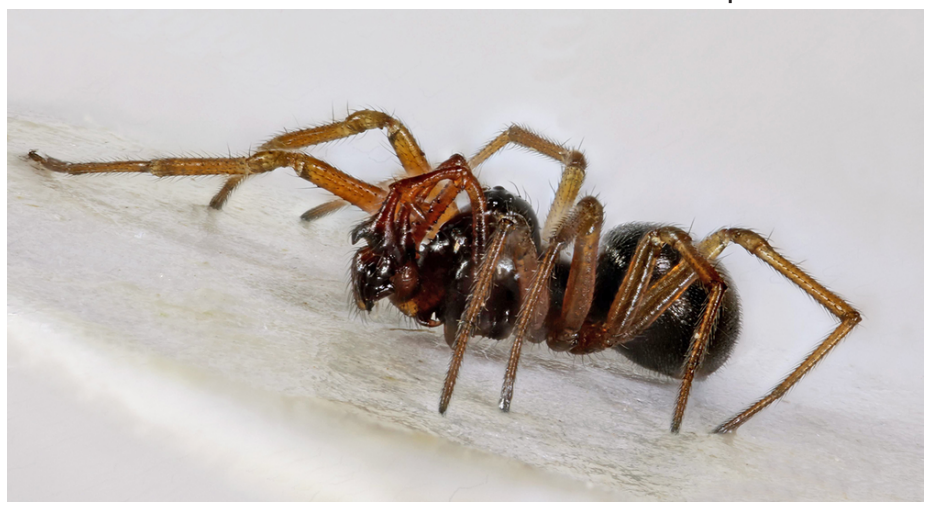

Wingless flier. Erigone atra spider from North Wales, UK. Credit: J. Graham (CC BY 2.0)

mechanics problem with creativity and care." Regarding the multithread dynamics, the researchers "demonstrate rigorously a conclusion that is intuitively simple yet challenging to prove."

The team also examined two different ways by which electric charge could be distributed on a thread. They performed the simulations assuming either a uniform distribution along the entire thread or a distribution localized at the thread's tip. Those studies showed that both could generate the force necessary for lifting. Going beyond biology, Habchi says that the work could lead to new devices for environmental science. "This understanding could be helpful for designing new types of ballooning sensors as probes to explore atmospheric properties."

Rachel Berkowitz is a Corresponding Editor for Physics based in Vancouver, Canada.

\section{REFERENCES}

1. C. Habchi and M. K. Jawed, "Ballooning in spiders using multiple silk threads," Phys. Rev. E 105, 034401 (2022). 